\title{
Environmental and occupational exposure to chromium in Iran: A systematic review
}

\author{
Leila Tavakkoli ${ }^{1}$, Zahra Zamani Nasab ${ }^{1}$, Narges Khanjani*2 \\ ${ }^{1}$ Department of Epidemiology and Biostatistics, School of Public Health, Kerman University of Medical Sciences, Kerman, Iran \\ ${ }^{2}$ Environmental Health Engineering Research Center, Kerman University of Medical Sciences, Kerman, Iran
}

Received: April 13, 2017

DOI: $10.5430 /$ jer.v3n2p31
Accepted: June 5, 2017

Online Published: July 3, 2017

URL: https://doi.org/10.5430/jer.v3n2p31

\begin{abstract}
Background: Chromium $(\mathrm{Cr})$ is a heavy metal and trace mineral found in various forms. $\mathrm{Cr}$ III is an essential nutrient, that is normally found in human blood and urine; in contrast, Cr VI is listed in group I carcinogens and is hazardous for human health. Several studies have been done about measuring human Chromium levels in Iran and this review attempted to summarize these studies.

Methods: Electronic resources including SID, Magiran, PubMed, Web of Knowledge, and Google Scholar were searched; with phrases including "Exposure to Chromium, Chromium exposure, Chromium measuring, Occupational exposure to Chromium, Environmental exposure to Chromium, Chromium" and their Persian translations until December 2, 2016.

Results: From the 1,309 retrieved articles, 32 articles were selected. In welding, electroplating and cement companies, chromium concentrations in workers who dealt directly with $\mathrm{Cr}$ were higher than others employees. In the general population, people who lived near a factory or an industrial center, or people who had taken dental treatments especially with orthodontic appliances, had higher Cr levels. Cr deficiency was also investigated in several studies, for its possible relation to diabetes type 2 .

Conclusion: Cr VI exposure should be under surveillance in Iranian industrial workers; and residential areas should be kept in a safe distance from $\mathrm{Cr}$ producing industries. Cr III deficiency might be related to diabetes type 2 .
\end{abstract}

Key Words: Chromium, Environmental exposures, Heavy metals, Iran

\section{INTRODUCTION}

Chromium (Cr) is a shiny and silvery-gray, brittle and hard metal, that is resistant to oxidation, even at high temperatures. ${ }^{[1,2]} \mathrm{Cr}$ can be naturally found in rocks, plants and soil,${ }^{[3]}$ and from a public health perspective, it is one of the toxic heavy metals. ${ }^{[4]}$ According to an Agency for Toxic Substances and Diseases Registry (ATSDR) report, in the US environment, the median concentrations of $\mathrm{Cr}$ in ambient air is $<20 \mathrm{ng} / \mathrm{m}^{3}$ and for indoor air with smoking it can be 100-400 times higher. The mean level of $\mathrm{Cr}$ in soil is $37.0 \mathrm{mg} / \mathrm{kg}$, and in drinking water is less than $5 \mu \mathrm{g} / \mathrm{L}^{[3]}$
$\mathrm{Cr}$ can be found in various forms $\mathrm{Cr}$ (II) to $\mathrm{Cr}$ (IV) and it is stable in the form of $\mathrm{Cr}$ III. ${ }^{[4]} \mathrm{Cr}$ VI is listed in group 1 carcinogens by International Agency for Research on Cancer $\left(\right.$ IARC) ${ }^{[5]}$ and is a human carcinogen. ${ }^{[6]} \mathrm{Cr}$ (III) is an essential nutrient ${ }^{[7]}$ that is normally found in human blood and urine $^{[3]}$ and is classified by IARC ${ }^{[5]}$ in group 3 , which means there is no sufficient evidence about its carcinogenesis. ${ }^{[6]}$ The Institute of Medicine of the National Academy of Sciences considers, 20-45 $\mu \mathrm{g}$ of Cr III intake ${ }^{[3]}$ (21-25 micrograms per day for women and 25-35 micrograms per day for men), ${ }^{[8]}$ adequate and enough for adults and adolescents. ${ }^{[3]} \mathrm{Cr}$ III

\footnotetext{
* Correspondence: Narges Khanjani; Email: n_khanjani@kmu.ac.ir; Address: Environmental Health Engineering Research Center, Kerman University of Medical Sciences, Kerman, Iran.
} 
deficiency may cause, heart diseases, metabolic disorders and diabetes, and its high intake can cause skin irritation. ${ }^{[1]}$

The general population may be exposed to $\mathrm{Cr}$ through breathing, eating and drinking food or water and skin contact. ${ }^{[1]}$ ATSDR reported that, in the US general population, the mean concentration of $\mathrm{Cr}$ in human serum and urine is, $0.16-0.10 \mu \mathrm{g} / \mathrm{L}$ and $0.22 \mu \mathrm{g} / \mathrm{L}$, respectively. ${ }^{[3]} \mathrm{Cr}$ is widely used in various industries, including plating, welding, dyes and pigments, leather and wood products. ${ }^{[1-4]}$ According to the report of Occupational Safety and Health Administration (OSHA), in general and in construction industries, the permissible air borne exposure limit (PEL) based on timeweighted average (TWA) for $\mathrm{Cr}$ II, Cr III and $\mathrm{Cr}$ VI is 0.5 $\mathrm{mg} / \mathrm{m}^{3}, 0.5 \mathrm{mg} / \mathrm{m}^{3}$ and $1 \mathrm{mg} / \mathrm{m}^{3}$, respectively. ${ }^{[9]}$

Cr VI is hazardous for human health and can cause health problems including, respiratory complications (lung irritation, nasal and lung problems), digestive complications (inflammation and ulceration of the stomach and small bowel lesions), hematological complications (anemia hypochromic and microcytic) as well as adverse reproductive effects (reducing sperm count and epididymal damages). ${ }^{[3]}$

Several studies have been performed, in different groups and cities in Iran about human levels of Chromium. This paper attempts to summarize these studies and make an overall estimation about the situation of this contaminant in humans, in Iran.

\section{MethodS}

\subsection{Databases and searching strategy}

Iranian/Persian electronic resources including SID, Magiran and international databases including PubMed, Web of Knowledge and Google Scholar were searched; with phrases including "Exposure to Chromium, Chromium exposure,
Chromium measuring, Occupational exposure to Chromium, Environmental exposure to Chromium, Chromium" and their Persian translations until December 2, 2016.

\subsection{Data extraction and Inclusion criteria}

All articles were reviewed by two authors separately. Among them, studies that investigated occupational or environmental exposures to Chromium in Iran and measured Chromium concentration in human tissues, were included in this study.

Studies done outside Iran, those that were not performed on humans, or did not report an estimate for chromium contamination in humans were excluded. Therefore, articles which measured chromium levels in animals, plants, air (including workers breathing zone), water and soil were excluded.

Information including first author, year of data collection, population, sample size, mean and standard deviation of Cr levels, location of sampling and $p$-values of subgroup comparisons, were retrieved from each article.

\section{RESUlts}

Finally, 32 articles were selected from 1,309 retrieved articles. The population under study in these articles and the method of reporting was very heterogeneous across studies and therefore pooled results were not calculated. The process of article selection has been shown in Figure 1.

Studies except one ${ }^{[10]}$ showed that people who had taken dental treatments especially orthodontic appliances for some time, had higher $\mathrm{Cr}$ levels in their body tissues than others (see Table 1).

All studies except one ${ }^{[21]}$ showed that $\mathrm{Cr}$ concentrations in patients with diabetes type 2 was lower than healthy controls and suggested a probable relation between $\mathrm{Cr}$ deficiency and diabetes (see Table 2).

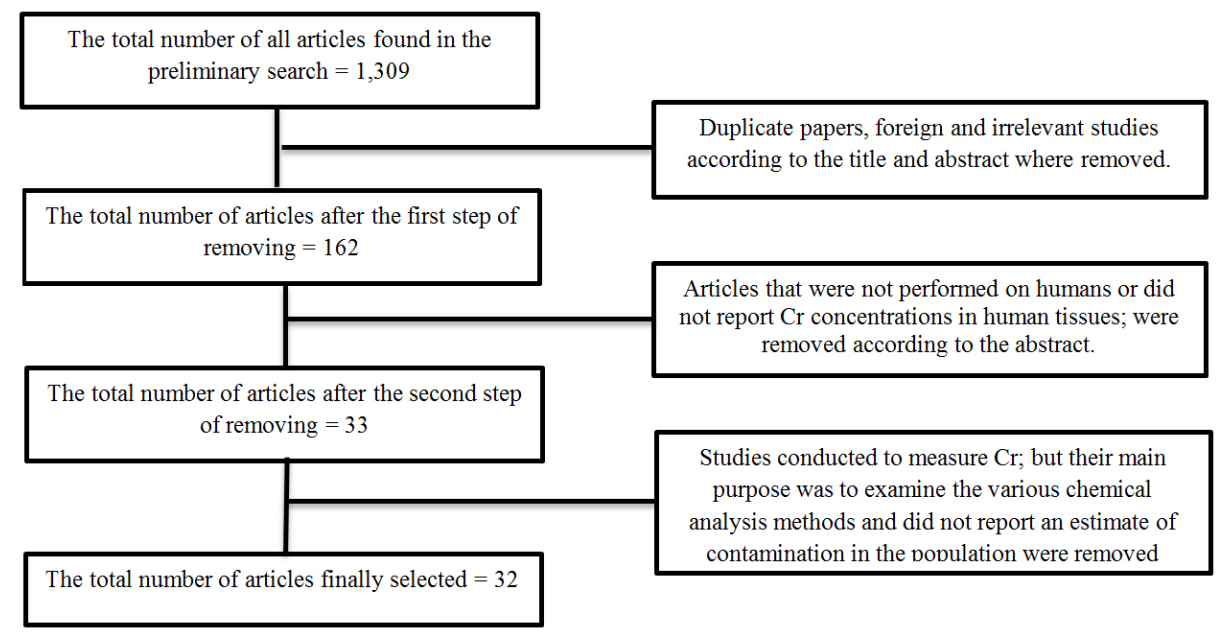

Figure 1. Process of article selection 
Table 1. Summary of studies measuring $\mathrm{Cr}$ in orthodontic patients in Iran

\begin{tabular}{|c|c|c|c|c|c|c|}
\hline & $\begin{array}{l}\text { First Author } \\
\text { and year of data } \\
\text { collection (Ref) }\end{array}$ & Population & Location & Sample & Mean \pm SD of $\mathbf{C r}$ & $\begin{array}{l}p \text {-value of } \\
\text { comparison }\end{array}$ \\
\hline 1 & $\begin{array}{l}\text { Amini, } \\
\text { 2013-2014 }\end{array}$ & $\begin{array}{l}30 \text { orthodontic patients } 11-26 \\
\text { years old: The patients were } \\
\text { divided into two groups of } \\
\text { experimental (metal-injection } \\
\text { molding (MIM) brackets) and } \\
\text { control (Conventional } \\
\text { brackets), } \mathrm{n}=15 \text { in each group. }\end{array}$ & $\begin{array}{l}\text { Department of } \\
\text { Orthodontics, } \\
\text { Dental Branch, } \\
\text { Islamic Azad } \\
\text { University, Tehran }\end{array}$ & Saliva & $\begin{array}{l}\text { Brackets used: } \\
\text { Baseline (pretreatment): } \\
\text {-Controls }=0.25 \pm 0.56 \mu \mathrm{g} / 1 \\
\text {-MIM brackets }=0.42 \pm 0.48 \mu \mathrm{g} / \mathrm{l} \\
60^{\text {th }} \text { day: } \\
- \text { Controls }=0.35 \pm 00.62 \mu \mathrm{g} / 1 \\
\text {-MIM brackets }=0.26 \pm 0.57 \mu \mathrm{g} / 1 \text { (decreased) } \\
\text { Regardless of the brackets used: } \\
\text { Baseline (pretreatment) }=0.34 \pm 0.52 \mu \mathrm{g} / 1 \\
60^{\text {th }} \text { day }=0.3 \pm 0.59 \mu \mathrm{g} / 1\end{array}$ & $\begin{array}{l}\text { In MIM brackets }= \\
.0438 \text { but } \downarrow \\
.110\end{array}$ \\
\hline 2 & $\begin{array}{l}\text { Amini, } \\
\text { 2013-2014 }\end{array}$ & $\begin{array}{l}24 \text { patients with orthodontics } \\
\text { (12 males and } 12 \text { females) }\end{array}$ & $\begin{array}{l}\text { The Orthodontics } \\
\text { Department of Azad } \\
\text { University, Tehran }\end{array}$ & Hair & $\begin{array}{l}\text { Baseline: } \\
\text { Females }=0.1440 \pm 0.0760 \mu \mathrm{g} / \mathrm{g} \\
\text { Males }=0.1469 \pm 0.0812 \mu \mathrm{g} / \mathrm{g} \\
\text { Total }=0.1455 \pm 0.0769 \mu \mathrm{g} / \mathrm{g} \\
\\
6 \text { months later: } \\
\text { Females }=0.1652 \pm 0.0692 \mu \mathrm{g} / \mathrm{g} \\
\text { Males }=0.1713 \pm 0.0752 \mu \mathrm{g} / \mathrm{g} \\
\text { Total }=0.1683 \pm 0.0707 \mu \mathrm{g} / \mathrm{g} \\
\\
\text { Increase in Cr in all patients happened } 6 \text { months after } \\
\text { orthodontic treatment }=0.0229 \pm 0.0266 \mu \mathrm{g} / \mathrm{g}\end{array}$ & .0002 \\
\hline 3 & $\begin{array}{l}\text { Amini, the } \\
\text { sampling was } \\
\text { performed } 16 \pm 2 \\
\text { months after the } \\
\text { start of treatment } \\
\text { with fixed } \\
\text { orthodontic } \\
\text { appliances, year } \\
\text { of data collection }_{\text {not reported }}^{[12]}\end{array}$ & $\begin{array}{l}56 \text { Subjects, } 28 \text { subjects with } \\
\text { fixed appliances in both arches } \\
\text { ( } 16 \text { females and } 12 \text { males) and } \\
28 \text { controls (a same-gender } \\
\text { sister or brother) without any } \\
\text { orthodontic appliance }\end{array}$ & Tehran & Saliva & $\begin{array}{l}\text { With appliance }=2.6 \pm 1.6 \mathrm{ng} / \mathrm{ml} \\
\text { Without appliance }=2.2 \pm 1.8 \mathrm{ng} / \mathrm{ml}\end{array}$ & .03 \\
\hline 4 & $\begin{array}{l}\text { Amini, } \\
\text { 2011-2012 }\end{array}$ & $\begin{array}{l}24 \text { patients with only the } \\
\text { maxillary arch stainless steel } \\
\text { brackets treatment ( } 12 \text { male } \\
\text { and } 12 \text { female) }\end{array}$ & $\begin{array}{l}\text { Department of } \\
\text { Orthodontics, } \\
\text { Dental Branch, } \\
\text { Islamic Azad } \\
\text { University, Tehran }\end{array}$ & $\begin{array}{l}\text { Gingival } \\
\text { Crevicul } \\
\text { ar Fluid } \\
(\mathrm{GCF})\end{array}$ & $\begin{array}{l}\text { Pretreatment (baseline) }=1.978 \pm 0.721 \mathrm{mg} / \mathrm{g} \text { of GCF } \\
1 \mathrm{month} \text { after the initiation of treatment }=4.135 \pm 1.591 \\
\mathrm{mg} / \mathrm{g} \text { of GCF } \\
6 \text { month after baseline }=13.760 \pm 3.555 \mathrm{mg} / \mathrm{g} \text { of GCF }\end{array}$ & $\begin{array}{l}\text { Baseline vs Month } \\
\text { one }<.001 \\
\text { Baseline vs Month } \\
\text { six }<.001\end{array}$ \\
\hline 5 & $\begin{array}{l}\text { Khane Masjedi, } \\
2014-2015^{[14]}\end{array}$ & $\begin{array}{l}24 \text { female and } 22 \text { males fixed } \\
\text { orthodontic patients: } \\
\text { Conventional brackets } \\
\text { (control) and Metal injection } \\
\text { molding (MIM) brackets } \\
\text { (experimental) (A total of } 60 \\
\text { patients were assessed until } 46 \\
\text { patients were included) }\end{array}$ & $\begin{array}{l}\text { Orthodontics } \\
\text { Department of } \\
\text { Ahvaz University of } \\
\text { Medical Sciences, } \\
\text { Ahvaz }\end{array}$ & Hair & $\begin{array}{l}\text { Before treatment }=0.1657 \pm 0.0884 \mu \mathrm{g} / \mathrm{g} \\
\text { After } 6 \text { months }=0.3066 \pm 0.1362 \mu \mathrm{g} / \mathrm{g} \\
\text { Difference }=0.1409 \pm 0.1131 \mu \mathrm{g} / \mathrm{g}\end{array}$ & .305 \\
\hline \multirow[t]{2}{*}{6} & \multirow[t]{2}{*}{$\begin{array}{l}\text { Amini, year of } \\
\text { data collection } \\
\text { not reported }\end{array}$} & \multirow[t]{2}{*}{$\begin{array}{l}20 \text { patents with fixed } \\
\text { orthodontics ( } 12 \text { female and } 8 \\
\text { male) }\end{array}$} & \multirow[t]{2}{*}{$\begin{array}{l}\text { The Orthodontics } \\
\text { Department of Azad } \\
\text { University, Tehran }\end{array}$} & \multirow[t]{2}{*}{ Saliva } & $\begin{array}{l}\text { Immediately before initiation of treatment (baseline) }= \\
3.86 \pm 1.34 \mu \mathrm{g} / \mathrm{L} \\
6 \text { months after orthodontic treatment }=4.60 \pm 6.11 \mu \mathrm{g} / \mathrm{L}\end{array}$ & $\begin{array}{l}\text { Baseline vs Month } \\
6=.360 \\
\text { Baseline vs Month } \\
12=.0038\end{array}$ \\
\hline & & & & & 12 month after orthodontic treatment $=2.04 \pm 1.66 \mu \mathrm{g} / \mathrm{L}$ & $\begin{array}{l}\text { Month 6-Month } \\
12=.046\end{array}$ \\
\hline 7 & $\begin{array}{l}\text { Amini, year of } \\
\text { data collection } \\
\text { not reported } \\
\text { (during } 3 \text { years) } \\
{[16]}\end{array}$ & $\begin{array}{l}60 \text { dental patients }(30 \text { cases } \\
\text { with fixed orthodontic } \\
\text { appliances and } 30 \text { controls } \\
\text { without any type of orthodontic } \\
\text { appliances or metal restoration } \\
\text { in their mouth }\end{array}$ & $\begin{array}{l}\text { The Orthodontics } \\
\text { Department of Azad } \\
\text { University, Tehran }\end{array}$ & $\begin{array}{l}\text { Mucosa } \\
\text { cell }\end{array}$ & $\begin{array}{l}\text { Cases }=4.24 \pm 1.82 \mathrm{ng} / \mathrm{mg} \\
\text { Controls }=3.46 \pm 1.65 \mathrm{ng} / \mathrm{mg}\end{array}$ & .09 \\
\hline 8 & $\begin{array}{l}\text { Arash, year of } \\
\text { data collection } \\
\text { not reported* }{ }^{17]}\end{array}$ & $\begin{array}{l}11 \text { fixed orthodontic patients ( } 7 \\
\text { females and } 4 \text { males) }\end{array}$ & Babol & Saliva & $\begin{array}{l}\text { Before treatment }=0.483 \pm 0.324 \mathrm{mg} / \mathrm{dl} \\
1 \text { day later }=0.471 \pm 0.238 \mathrm{mg} / \mathrm{dl} \\
1 \text { week later }=0.685 \pm 0.624 \mathrm{mg} / \mathrm{dl} \\
1 \text { month later }=0.741 \pm 0.414 \mathrm{mg} / \mathrm{dl} \\
2 \text { months later }=1.240 \pm 1.244 \mathrm{mg} / \mathrm{dl} \\
6 \text { months later }=3.244 \pm 0.777 \mathrm{mg} / \mathrm{dl}\end{array}$ & .013 \\
\hline 9 & $\begin{array}{l}\text { Yassaei, year of } \\
\text { data collection } \\
\text { not reported }^{[18]}\end{array}$ & $\begin{array}{l}32 \text { patients } 11 \text { to } 24 \text { years old } \\
\text { who visited the orthodontic } \\
\text { clinic }\end{array}$ & Yazd & Saliva & $\begin{array}{l}\text { T1: before appliance placement }=2.6 \pm 3.14 \mu \mathrm{g} / \mathrm{L} \\
\text { T2: } 20 \text { days after appliance placement }=3.68 \pm 3.95 \mu \mathrm{g} / \mathrm{L} \\
\text { T3: } 3 \text { months after appliance placement }=3.41 \pm 3.36 \mu \mathrm{g} / \mathrm{L} \\
\text { T4: } 6 \text { months after appliance placement }=3.39 \pm 3.41 \mu \mathrm{g} / \mathrm{L}\end{array}$ & .168 \\
\hline 10 & Amini, $2012^{[19]}$ & $\begin{array}{l}30 \text { patients with fixed } \\
\text { orthodontics }\end{array}$ & $\begin{array}{l}\text { The Orthodontics } \\
\text { Department of Azad } \\
\text { University, Tehran }\end{array}$ & Saliva & $\begin{array}{l}\text { 1st time (before orthodontics) }=4.1 \pm 2.3 \mu \mathrm{g} / \mathrm{L} \\
\text { 2nd time }(3 \text { months later })=4.4 \pm 3.2 \\
3 \mathrm{rd} \text { time }(5 \text { min following the induction of stress })=4.8 \pm \\
3.3 \mu \mathrm{g} / \mathrm{L} \\
4 \text { th time }(30 \text { min following } \\
\text { the induction of stress })=5.1 \pm 3.3 \mu \mathrm{g} / \mathrm{L}\end{array}$ & $\begin{array}{l}\text { The mean amount } \\
\text { of salivary Cr was } \\
\text { not significantly } \\
\text { different between } \\
\text { the four time } \\
\text { points }(p>.05)\end{array}$ \\
\hline 11 & $\begin{array}{l}\text { Amini, } \\
2012-2013^{[20]}\end{array}$ & $\begin{array}{l}10 \text { patients with fixed } \\
\text { orthodontics }\end{array}$ & $\begin{array}{l}\text { The Orthodontics } \\
\text { Department of the } \\
\text { Azad University, } \\
\text { Tehran }\end{array}$ & Saliva & $\begin{array}{l}\text { First time (before orthodontics) }=5.2 \pm 3.38 \mu \mathrm{g} / \mathrm{L} \\
\text { Second time }(3 \text { months later and before stress) }=5.42 \pm \\
2.44 \mu \mathrm{g} / \mathrm{L} \\
\text { Third time (after stress) }=6.27 \pm 2.68 \mu \mathrm{g} / \mathrm{L}\end{array}$ & $>.05$ \\
\hline
\end{tabular}


Table 2. Studies measuring $\mathrm{Cr}$ in diabetic patients and their controls in Iran

\begin{tabular}{|c|c|c|c|c|c|c|}
\hline & $\begin{array}{l}\text { First Author and } \\
\text { year of data } \\
\text { collection (Ref) }\end{array}$ & Population & Location & Sample & Mean \pm SD of $\mathbf{C r}$ & $p$-value \\
\hline 12 & $\begin{array}{l}\text { Nourmohammadi, } \\
\text { year of data } \\
\text { collection not } \\
\text { reported }^{[21]}\end{array}$ & $\begin{array}{l}40 \text { subjects ( } 20 \text { diabetic patients who attended } \\
\text { the diabetic clinic and were taking insulin and } \\
20 \text { controls from university staff with no } \\
\text { complications and not receiving any } \\
\text { medication) }\end{array}$ & Semnan & Hair & $\begin{array}{l}\text { Diabetic Patients }=2.418 \pm 2.089 \mu \mathrm{g} / \mathrm{g} \\
\text { Controls }=0.3625 \pm 0.23 \mu \mathrm{g} / \mathrm{g}\end{array}$ & .001 \\
\hline 13 & $\begin{array}{l}\text { Basaki, year of data } \\
\text { collection not } \\
\text { reported }{ }^{[22]}\end{array}$ & $\begin{array}{l}40 \text { female subjects referred to Dr. Saeb's } \\
\text { specialized hormone lab ( } 20 \text { patients with } \\
\text { type } 2 \text { diabetes and } 20 \text { nondiabetics doing a } \\
\text { checkup) }\end{array}$ & Shiraz & $\begin{array}{l}\text { Blood } \\
\text { (Serum) }\end{array}$ & $\begin{array}{l}\text { Nondiabetic controls }=0.27 \pm 0.15 \text { (unit?) } \\
\text { Patients with type } 2 \text { diabetes }=0.16 \pm 0.07 \text { (unit?)* }\end{array}$ & $<.05$ \\
\hline 14 & Rafiei, $2012^{[23]}$ & $\begin{array}{l}132 \text { pre-diabetic patients (but } 24 \text { patients } \\
\text { were not included in the final statistical } \\
\text { analyses because they did not complete their } \\
\text { laboratory studies) }\end{array}$ & $\begin{array}{l}\text { Shariati } \\
\text { Hospital, } \\
\text { Isfahan }\end{array}$ & Blood & $\begin{array}{l}\text { The mean and standard deviation of serum } \mathrm{Cr} \text { was } \\
1.08 \pm 1.06 \mu \mathrm{g} / \mathrm{L} \text {. } \\
\text { Thirty-four }(31.5 \%) \text { patients had Cr deficiency and } \\
74(68.5 \%) \text { patients had normal } \\
\text { Cr. } \\
\text { There was no significant difference in Cr between } \\
\text { men and women, between the investigated age } \\
\text { groups ( }<50 \text { years and } \geq 50 \text { years), and between } \\
\text { patients with and without a family history of } \\
\text { diabetes. } \\
\text { No significant differences in age, BMI, FBS or } \\
\text { insulin were observed between Cr deficiency and } \\
\text { Cr normal groups. } \\
\text { In the group with a normal level of Cr, there was a } \\
\text { significant reversed correlation between Cr levels } \\
\text { and age. }(r=-0.276)\end{array}$ & $>.05$ \\
\hline 15 & $\begin{array}{l}\text { Saburi, } \\
2008-2009^{[24]}\end{array}$ & $\begin{array}{l}30 \text { type } 2 \text { diabetes patients referred to Sina } \\
\text { Hospital ( } 15 \text { males and } 15 \text { females) and } 30 \\
\text { healthy individuals }\end{array}$ & Tabriz & $\begin{array}{l}\text { Blood } \\
\text { (Serum) }\end{array}$ & $\begin{array}{l}\text { Diabetic Patients }=0.80 \pm 0.28 \mu \mathrm{g} / \mathrm{dl} \\
\text { Controls }=1.19 \pm 0.33 \mu \mathrm{g} / \mathrm{dl}\end{array}$ & $<.001$ \\
\hline 16 & $\begin{array}{l}\text { Palizban, year of } \\
\text { data collection not } \\
\text { reported }^{[25]}\end{array}$ & $\begin{array}{l}-47 \text { patients with both metabolic syndrome } \\
\text { and type } 2 \text { diabetes ( } 30 \text { females and } 17 \text { males) } \\
\text { and } \\
-45 \text { patients with metabolic syndrome and } \\
\text { without type } 2 \text { diabetes ( } 33 \text { females and } 12 \\
\text { males) } \\
\text { and } \\
-35 \text { healthy individuals as a control group ( } 23 \\
\text { females and } 12 \text { males) }\end{array}$ & Isfahan & Blood & $\begin{array}{l}\text { Patients with both metabolic syndrome and type } 2 \\
\text { diabetes: } \\
11.60 \pm 14.73 \mu \mathrm{g} / \mathrm{L} \\
\text { Patients with metabolic syndrome and without } \\
\text { type } 2 \text { diabetes: } \\
18.83 \pm 22.82 \mu \mathrm{g} / \mathrm{L} \\
\text { Control: } \\
28.02 \pm 19.28 \mu \mathrm{g} / \mathrm{L}\end{array}$ & $\begin{array}{l}p \text {-value for } \\
\text { one way } \\
\text { ANOVA }= \\
.002\end{array}$ \\
\hline 17 & $\begin{array}{l}\text { Parsaeian, year of } \\
\text { data collection not } \\
\text { reported }^{[26]}\end{array}$ & $\begin{array}{l}370 \text { participants: } 108 \text { patients with type } 2 \\
\text { diabetes ( } 57 \text { males and } 51 \text { females) and } 47 \\
\text { patients with type } 1 \text { diabetes ( } 21 \text { males and } 26 \\
\text { females) and also } 108 \text { individuals with (IGT) } \\
\text { impaired glucose tolerance ( } 56 \text { males and } 51 \\
\text { females) and } 108 \text { controls ( } 54 \text { males and } 54 \\
\text { females) }\end{array}$ & Yazd & $\begin{array}{l}\text { Blood } \\
\text { (Serum) }\end{array}$ & $\begin{array}{l}\text { Controls: } \\
\text {-Total }=0.24 \pm 0.023 \mu \mathrm{g} / \mathrm{L} \\
\text { Impaired glucose tolerance }(\mathrm{IGT}) \text { : } \\
\text {-Total }=0.17 \pm 0.025 \mu \mathrm{g} / \mathrm{L} \\
\text { Type } 1 \text { diabetes: } \\
\text {-Total }=0.090 \pm 0.028 \mu \mathrm{g} / \mathrm{L} \\
\text { Type } 2 \text { diabetes: } \\
\text {-Total }=0.097 \pm 0.021 \mu \mathrm{g} / \mathrm{L}\end{array}$ & $\begin{array}{l}p \text {-value for } \\
\text { one way } \\
\text { ANOVA }= \\
.004\end{array}$ \\
\hline 18 & $\begin{array}{l}\text { Tadayon, } \\
2009-2010\end{array}$ & $\begin{array}{l}250 \text { women } 33-35 \text { years old: } 142 \text { controls and } \\
108 \text { diabetes patients type } 2\end{array}$ & Tehran & Hair & $\begin{array}{l}\text { Patients }=0.777 \pm 1.8 \mu \mathrm{g} / \mathrm{g} \\
\text { Controls }=1.505 \pm 1.33 \mu \mathrm{g} / \mathrm{g}\end{array}$ & $\begin{array}{l}p \text {-value not } \\
\text { reported }\end{array}$ \\
\hline 19 & $\begin{array}{l}\text { Abdolsamadi, } \\
2009-2011^{[28]}\end{array}$ & $\begin{array}{l}70 \text { pregnant women: } 35 \text { gestational diabetes } \\
\text { mellitus and } 35 \text { non-diabetic pregnant women }\end{array}$ & Hamadan & Saliva & $\begin{array}{l}\text { Patients }=6.63 \pm 5.48 \mu \mathrm{g} / 100 \mathrm{ml} \\
\text { Controls }=8.30 \pm 6.95 \mu \mathrm{g} / 100 \mathrm{ml}\end{array}$ & .001 \\
\hline 20 & $\begin{array}{l}\text { Nasli-Esfahani, year } \\
\text { of data collection } \\
\text { not reported }\end{array}$ & $\begin{array}{l}301 \text { participants: diabetic patients }(n=150) \\
\text { and healthy controls }(n=151) \text {. }\end{array}$ & $\begin{array}{l}\text { Dr Shariati } \\
\text { Hospital, } \\
\text { Tehran }\end{array}$ & $\begin{array}{l}\text { Hair } \\
\text { Urine } \\
\text { Nail } \\
\text { Serum }\end{array}$ & $\begin{array}{l}\text { Hair: } \\
\text {-Patients }=1.21 \pm 0.97 \mu \mathrm{g} / \mathrm{g} \\
\text {-Control }=4.36 \pm 1.03 \mu \mathrm{g} / \mathrm{g} \\
\text { Urine: } \\
\text {-Patients }=0.010 \pm 0.006 \mu \mathrm{g} / \mathrm{ml} \\
\text {-Control= } 0.009 \pm 0.007 \mu \mathrm{g} / \mathrm{ml} \\
\text { Nail: } \\
\text {-Patients }=1.10 \pm 0.34 \mu \mathrm{g} / \mathrm{g} \\
\text {-Control }=2.37 \pm 0.64 \mu \mathrm{g} / \mathrm{g} \\
\text { Serum: } \\
\text {-Patients }=0.0013 \pm 0.0004 \mu \mathrm{g} / \mathrm{ml} \\
\text {-Control }=0.0018 \pm 0.0014 \mu \mathrm{g} / \mathrm{ml}\end{array}$ & $\begin{array}{l}<.05 \\
>.05 \\
<.05 \\
<.05\end{array}$ \\
\hline 21 & $\begin{array}{l}\text { Yousefi Rad, year } \\
\text { of data collection } \\
\text { not reported }{ }^{[30]}\end{array}$ & $\begin{array}{l}30 \text { ( } 15 \text { male and } 15 \text { female) diabetes patients } \\
\text { and } 30 \text { ( } 15 \text { male and } 15 \text { female) healthy } \\
\text { people }\end{array}$ & $\begin{array}{l}\text { Sina Hospital, } \\
\text { Tabriz }\end{array}$ & Serum & $\begin{array}{l}\text { Diabetes patients }=0.80 \pm 0.258 \mu \mathrm{g} / \mathrm{dl} \\
\text { Healthy people }=1.19 \pm 0.33 \mu \mathrm{g} / \mathrm{dl}\end{array}$ & $<0.001$ \\
\hline 22 & $\begin{array}{l}\text { Makhlough, } \\
2010-2011^{[31]}\end{array}$ & $\begin{array}{l}70 \text { patients with type } 2 \text { diabetic nephropathy } \\
\text { (macro and micro-albuminuria) and } 70 \\
\text { healthy individuals }\end{array}$ & $\begin{array}{l}\text { Sari, } \\
\text { Mazandaran }\end{array}$ & $\begin{array}{l}\text { Blood } \\
\text { (Serum) }\end{array}$ & $\begin{array}{l}\text { Approximate values based on Figure } 1 \text { are: } \\
\text { Cases: Patients with diabetic nephropathy: } \\
\mathrm{Cr}=0.28 \mu \mathrm{gr} / \mathrm{L} \\
\text { Controls: Healthy Group: } \\
\mathrm{Cr}=0.6 \mu \mathrm{gr} / \mathrm{L} \\
\text { Approximate values based on figure } 2 \text { are: } \\
\text { Cases with Micro Albuminuria: } \\
\mathrm{Cr}=0.28 \mu \mathrm{gr} / \mathrm{L} \\
\mathrm{Cases} \text { with Macro Albuminuria: } \\
\mathrm{Cr}=0.27 \mu \mathrm{gr} / \mathrm{L}\end{array}$ & $\begin{array}{l}\text { Cases vs. } \\
\text { Controls } \\
<.001 \\
\\
\text { Comparing } \\
\text { cases with } \\
\text { macro vs. } \\
\text { micro } \\
\text { Albuminuria } \\
>.05\end{array}$ \\
\hline 23 & $\begin{array}{l}\text { Tadayon, year of } \\
\text { data collection not } \\
\text { reported }^{[32]}\end{array}$ & 100 women between 35 to 70 years of age & Tehran & Hair & $\begin{array}{l}\text { Approximate values based on Figure } 2 \text { are: } \\
\text { Diabetic patients }=0.6 \mu \mathrm{g} / \mathrm{g} \\
\text { Healthy Group }=1.3 \mu \mathrm{g} / \mathrm{g}\end{array}$ & $<.05$ \\
\hline
\end{tabular}


In industrial jobs, such as welding, electroplating and cement companies, Cr VI concentrations were higher in workers who dealt directly with $\mathrm{Cr}$ than others employees of the same industry. In the general population, the $\mathrm{Cr}$ concentration was various in different groups. One study showed $\mathrm{Cr}$ levels of serum were significantly higher in bladder cancer cases than healthy controls ${ }^{[33]}$ and the other showed $\mathrm{Cr}$ was lower in tinnitus patients. ${ }^{[34]}$ Also, one study showed $\mathrm{Cr}$ was significantly higher in matured boys with normal weight than low weight (see Table 3). ${ }^{[35]}$

Table 3. Summary of studies measuring Cr exposure in industrial workers or other groups in Iran

\begin{tabular}{|c|c|c|c|c|c|c|}
\hline & $\begin{array}{l}\text { First Author and year } \\
\text { of data collection (Ref) }\end{array}$ & Population & Location & Sample & Mean \pm SD of $\mathbf{C r}$ & $\begin{array}{l}p \text {-value of } \\
\text { comparisons }\end{array}$ \\
\hline 24 & $\begin{array}{l}\text { Nazarpour, year of data } \\
\text { collection not reported }{ }^{[36]}\end{array}$ & $\begin{array}{l}100 \text { mothers attending clinics were } \\
\text { sampled four to eight weeks after delivery }\end{array}$ & Varamin & $\begin{array}{l}\text { Breast } \\
\text { milk }\end{array}$ & $3 \pm 2.7 \mu \mathrm{g} / \mathrm{ml}$ & --- \\
\hline 25 & $\begin{array}{l}\text { Hashemian, years of } \\
\text { enrollment 2004-2008 } \\
\text { (Golestan Cohort } \\
\text { Study) }{ }^{[37]}\end{array}$ & $\begin{array}{l}30 \text { nail samples from the participants of } \\
\text { Golestan Cohort Study (GCS) who had } \\
\text { died in accidents during the first } 7 \text { years of } \\
\text { follow-up }\end{array}$ & Golestan & Toenail & $0.25 \pm 0.63 \mu \mathrm{g} / \mathrm{g}$ & --- \\
\hline 26 & Eskandary, 1999-2000 ${ }^{[38]}$ & 30 head injury patients & $\begin{array}{l}\text { Bahonar } \\
\text { Hospital, } \\
\text { Kerman }\end{array}$ & $\begin{array}{l}\text { Blood } \\
\text { (Serum) }\end{array}$ & $\begin{array}{l}\text { Severe head injury patients }=1.42 \pm 0.23 \\
\mathrm{mg} / \mathrm{L} \\
\text { Moderate head injury patients }=1.58 \pm 0.23 \\
\mathrm{mg} / \mathrm{L} \\
\text { Mild head injury patients }=1.41 \pm 0.2 \mathrm{mg} / \mathrm{L}\end{array}$ & $\begin{array}{l}\text { the concentration } \\
\text { of Cr in serum had } \\
\text { no significant } \\
\text { relation to the } \\
\text { degree of head } \\
\text { injury during the } \\
\text { first } 4 \text { hours }\end{array}$ \\
\hline 27 & $\begin{array}{l}\text { Alirezaee, year of data } \\
\text { collection not reported }^{[35]}\end{array}$ & $\begin{array}{l}45 \text { premature male pupils (primary } \\
\text { schools' children with average age of } 10 \\
\text { years) and } 49 \text { mature male pupils (high } \\
\text { school children with average age of } 17 \\
\text { years) were randomly selected from } 2 \\
\text { schools in the Fifth Education District of } \\
\text { Mashhad }\end{array}$ & Mashhad & Nail & $\begin{array}{l}\text { Premature: } \\
\text {-Normal weight }(\mathrm{n}=9)=0.00012 \pm 0.00001 \\
\text { percent } / 100 \text { gr nail } \\
\text {-Low weight }(\mathrm{n}=35)=0.00014 \pm 0.00001 \\
\text { percent } / 100 \text { gr nail } \\
\text { Matured: } \\
\text {-Normal weight }(\mathrm{n}=32)=0.00020 \pm 0.00002 \\
\text { percent } / 100 \text { gr nail } \\
\text {-Low weight }(\mathrm{n}=14)=0.00017 \pm 0.00003 \\
\text { percent } / 100 \text { gr nail }\end{array}$ & .043 \\
\hline 28 & Mazdak, 2006-2008 ${ }^{[33]}$ & $\begin{array}{l}51 \text { bladder cancer patients and } 58 \text { healthy } \\
\text { individuals }\end{array}$ & Isfahan & $\begin{array}{l}\text { Blood } \\
\text { (Serum) }\end{array}$ & $\begin{array}{l}\text { Patients }=128.82 \pm 16.99 \mu \mathrm{g} / \mathrm{L} \\
\text { Controls }=121.93 \pm 16.67 \mu \mathrm{g} / \mathrm{L}\end{array}$ & $<.05$ \\
\hline 29 & $\begin{array}{l}\text { Mozaffarinia, year not } \\
\text { reported }^{[34]}\end{array}$ & $\begin{array}{l}55 \text { patients with tinnitus and } 47 \text { healthy } \\
\text { volunteers }\end{array}$ & Isfahan & $\begin{array}{l}\text { Blood } \\
\text { (Serum) }\end{array}$ & $\begin{array}{l}\text { Patients }=26.71 \pm 5.39 \mu \mathrm{g} / \mathrm{dl} \\
\text { Controls }=30.57 \pm 4.75 \mu \mathrm{g} / \mathrm{dl} \\
\text { Male }=27.62 \pm 5.13 \mu \mathrm{g} / \mathrm{dl} \\
\text { Female }=29.40 \pm 5.58 \mu \mathrm{g} / \mathrm{dl}\end{array}$ & $\begin{array}{l}<.001 \\
.100\end{array}$ \\
\hline 30 & Golbabaei, $2011^{[39]}$ & $\begin{array}{l}119 \text { People ( } 94 \text { welders of natural gas } \\
\text { pipes, } 6 \text { back welders, } 29 \text { assistants and } 25 \\
\text { controls) }\end{array}$ & $\begin{array}{l}\text { Brujen } \\
\text { (Chaharma } \\
\text { hal and } \\
\text { Bakhtiari) }\end{array}$ & Urine & $\begin{array}{l}\text { Welders }=3.36 \pm 3.74 \mu \mathrm{g} / \mathrm{L} \\
\text { Back welders }=12.67 \pm 4.50 \mu \mathrm{g} / \mathrm{L} \\
\text { Assistants }=1.98 \pm 1.05 \mu \mathrm{g} / \mathrm{L} \\
\text { Control }=1.04 \pm 0.56 \mu \mathrm{g} / \mathrm{L}\end{array}$ & $\begin{array}{l}\text { Welders vs } \\
\text { Control }=0.004 \\
\text { Back welders vs } \\
\text { Control }<0.001\end{array}$ \\
\hline 31 & $\begin{array}{l}\text { Pournourmohammadi, } \\
\text { year of data collection not } \\
\text { reported }^{[40]}\end{array}$ & $\begin{array}{l}90 \text { volunteer male workers from a cement } \\
\text { factory }\end{array}$ & Darab, Fars & Serum & $\begin{array}{l}\text { Healthy control }(\mathrm{n}=30)=3.2 \pm 0.5 \mu \mathrm{g} / \mathrm{L} \\
\text { Indirect-exposed }(\mathrm{n}=28)=3.6 \pm 0.3 \mu \mathrm{g} / \mathrm{L} \\
\text { Direct-exposed }(\mathrm{n}=60)=5.2 \pm 0.4 \mu \mathrm{g} / \mathrm{L}\end{array}$ & $\begin{array}{l}\text { Directly exposed } \\
\text { vs Healthy } \\
\text { controls }=.009 \\
\text { Directly exposed } \\
\text { vs. Indirectly } \\
\text { exposed }=.009\end{array}$ \\
\hline 32 & $\begin{array}{l}\text { Golbabaei, year of data } \\
\text { collection not reported }^{[41]}\end{array}$ & $\begin{array}{l}45 \text { Chromium plating workers and } 40 \text { Zinc } \\
\text { plating workers as a control group }\end{array}$ & Isfahan & Urine & $\begin{array}{l}\text { Chromium plating workers: } \mathrm{Cr}=9.798 \pm 7.554 \\
\mu \mathrm{g} / \mathrm{g} \text { Creatinine } \\
\text { Zinc plating workers: } \\
\mathrm{Cr}=1.811 \pm 0.699 \mu \mathrm{g} / \mathrm{g} \text { Creatinine }\end{array}$ & $<.001$ \\
\hline
\end{tabular}

\section{Discussion}

Several studies have shown that $\mathrm{Cr}$ is involved in glucose and lipid metabolism and has antioxidant functions. ${ }^{[42]}$ Researchers think $\mathrm{Cr}$ improves glucose metabolism and insulin sensitivity in type two, type one and gestational diabetes, ${ }^{[43]}$ and it may play a small role in human weight control. ${ }^{[44]} \mathrm{It}$ is also an essential trace element for proper protein, carbohydrate and lipid metabolism. ${ }^{[8]}$ Studies have also shown associations between $\mathrm{Cr}$ deficiency and diabetes and cardio-

Published by Sciedu Press vascular diseases. $^{[45]}$

Some studies have mentioned that polycystic ovarian syndrome (PCOS) that may cause infertility in women, can be treated by using $\mathrm{Cr}$ supplements. ${ }^{[46,47]}$

Cr toxicity is mainly caused by the hexavalent form and can cause respiratory, dermal, cardiovascular, gastrointestinal, renal and carcinogenic effects. ${ }^{[48]}$ However, there is no clear evidence that shows acute inhalation of chromium or 
its compounds can cause immediate death. ${ }^{[49]}$

In Iran, many studies were performed to investigate and measure $\mathrm{Cr}$ concentration in water, soil, air, breathing zone of workers in related industries, or in food including rice, tea, cans, and also fishes and even in toys. Among the studies that evaluated $\mathrm{Cr}$ levels in human tissues in Iran, a large number of studies were performed in the general population and were more than the studies performed to investigate occupational exposures (29 from 32 articles). Also, one third of these studies were performed in the capital city of Iran, Tehran; and among patients who were under dental treatments especially with fixed orthodontic appliances (11 from 32 articles). Other studies were performed in big cities including Isfahan, Kerman, Yazd, Shiraz, Mashhad and Tabriz (8 from 32 articles) and in diabetes type 2 patients (11 from 32 articles).

\subsection{Environmental exposure to $\mathrm{Cr}$}

Chromium waste products are made during industrial process or mining. ${ }^{[50]}$ These toxic waste products can create vast environmental problems ${ }^{[50]}$ that affect human health by chromium toxicity and carcinogenicity. ${ }^{[51]}$ It seems necessary to run environmental monitoring programs by considering all routes of chromium exposure ${ }^{[51]}$ in Iran.

Higher than normal $\mathrm{Cr}$ exposure may result in elevated $\mathrm{Cr}$ levels in blood, urine, hair and nails. The most reliable tissues for measuring $\mathrm{Cr}$ exposure are blood and urine. ${ }^{[3]}$

Alirezaee et al. in Mashhad, investigated $\mathrm{Cr}$ concentrations in nails and showed that there was no significant relation between $\mathrm{Cr}$ concentration in nails before and after puberty in boys. ${ }^{[35]}$ Authors have commented that variations in trace elements intake such as $\mathrm{Cr}$ may affect growing during the premature and maturing periods. ${ }^{[35]}$ Also, it seems that chromium supplementation can increase muscle accretion (increasing muscle area and percentage of muscle) and fat loss. ${ }^{[52]}$

Nazarpour et al. in Varamin, showed that $\mathrm{Cr}$ concentration of breast milk were higher in women with a smoking partner and women who lived near a factory or an industrial area. ${ }^{[36]}$ The results of a study by Interdonato et al. in Italy also showed, adolescents living in the industrialized areas of northern Sicily had higher concentration of heavy metals including $\mathrm{Cr}$ in their body, although it was not significant. ${ }^{[53]}$

A case-control study from Isfahan, showed that $\mathrm{Cr}$ concentration in patients with bladder cancer was significantly higher than controls. ${ }^{[33]}$ Other studies have also shown a probable relation between exposure to $\mathrm{Cr}$ and lung cancer. ${ }^{[54]}$ The reaction of $\mathrm{Cr}$ VI with genetic matter can probably cause carcinogenicity ${ }^{[48,55]}$ and significant relations between exposure to $\mathrm{Cr}$ VI and lung and airways cancers have been reported. ${ }^{[48,55,56]}$
Oxidative stress may play a role in the pathogenesis of tinnitus. ${ }^{[54,57,58]}$ Meanwhile, Cr III has antioxidant effects ${ }^{[59,60]}$ and it seems that $\mathrm{Cr}$ III deficiency can cause tinnitus. Mozaffari Nia et al. in Isfahan showed a significant reduction in serum concentration of $\mathrm{Cr}$ in people with tinnitus compared to the control group. ${ }^{[34]}$

Chromium is a common component in the arch-wires of orthodontic appliances (fixed orthodontics) and is an element used in the alloy steel (17\%-22\%) used in fixed and conventional orthodontic appliances (brackets). ${ }^{[61]}$ Studies have shown that $\mathrm{Cr}$ concentrations have increased in hair samples, ${ }^{[11,14]}$ and in the Gingival Crevicular Fluid (GCF) of orthodontic patients. ${ }^{[13]}$ Therefore, a longer duration of treatment with fixed orthodontics may lead to increased $\mathrm{Cr}$ concentrations. The result of a study by A ğaoğlu et al. in Turkey also showed, fixed orthodontic appliances can significantly increase $\mathrm{Cr}$ concentration in blood and urine; but this increased did not exceed toxic levels. ${ }^{[62]}$ The results of a study by Singh et al. in India showed, $\mathrm{Cr}$ concentration of saliva after treatment with fixed orthodontic appliances significantly increased, especially in the first week after treatment. ${ }^{[63]}$ However, the results of the study by Kocadereli et al in Turkey showed, there was no significant difference between $\mathrm{Cr}$ concentration of saliva at baseline time (before treatment) and after two months of treatments in patients with fixed orthodontics. ${ }^{[64]}$

Nour Mohammadi et al. in Semnan showed that, $\mathrm{Cr}$ levels of hair samples in diabetes type 2 patients was significantly higher than controls (20 healthy volunteers). ${ }^{[21]}$ In contrast, studies from Tehran, ${ }^{[29,32]}$ Shiraz, and Tabriz, ${ }^{[22,24]}$ showed $\mathrm{Cr}$ in diabetic type 2 patients were significantly lower than controls. Likewise, Kazi et al. in Pakistan showed, Cr concentrations of hair and blood samples in diabetes type 2 patients were significantly lower than controls. ${ }^{[65]}$ Some researchers have stated that $\mathrm{Cr}$ is an essential element for the human body and necessary for insulin functioning and glucose metabolism. ${ }^{[43,66]}$ Palizban et al. in Isfahan showed, low $\mathrm{Cr}$ blood concentration was associated with diabetes and metabolic syndrome; and therefore suggested taking this element as a supplement. ${ }^{[25]}$ A study by Rafiei et al. in Isfahan showed that $\mathrm{Cr}$ deficiency was common in prediabetes patients and suggested that patients with diabetes and prediabetes should be screened for $\mathrm{Cr}$; and $\mathrm{Cr}$ deficiency should be eliminated in these patients. ${ }^{[23]}$

\subsection{Occupational exposure to $\mathrm{Cr}$}

Chromium can be released into the atmosphere through commercial and residential activities, such as combustion of gas, oil and coal or industrial activities including, chromium electroplating and steel production. ${ }^{[1-3]}$ About one third of the 
chromium released into atmosphere is Cr VI. ${ }^{[3]} \mathrm{A}$ considerable amount of $\mathrm{Cr}$ has been released to surface waters by electroplating, and the leather and textile industries. ${ }^{[3]}$

$\mathrm{Cr}$ VI is a toxic form of chromium and its compounds are used in many different industries as corrosion inhibitors, for production of pigments, metal finishing and plating, stainless steel production, leather tanning, and wood protection. ${ }^{[67,68]}$ Industrial employees can be exposed to Cr VI through inhaling airborne $\mathrm{Cr} \mathrm{VI}$ as dust, fume or mist during the different parts of production. ${ }^{[67]}$

Pour Nour Mohammadi et al. showed that $\mathrm{Cr}$ contamination was significantly higher in workers of a cement company in Darab who dealt with $\mathrm{Cr}$ directly, in comparison to administration employees in the same industry and controls. ${ }^{[40]}$ Another study by Golbabaei et al. in Borujen showed $\mathrm{Cr}$ VI concentration in the breathing zone and urine of welders who directly dealt with $\mathrm{Cr}$ was higher than controls. ${ }^{[39]}$ And in another study showed that $\mathrm{Cr}$ and $\mathrm{N}$-Acetylglucosamine (NAG) concentration in urine samples of $\mathrm{Cr}$ plating workers was significantly higher than plating workers. ${ }^{[41]} \mathrm{NAG}$ is an enzyme that initially originates in the lysosomes of tubular cells. This marker does not get filtered by the renal glomerular system ${ }^{[41]}$ and because of this, NAG may increase in subjects exposed to substances toxic for the renal tubular cells. In humans, its increased excretion is probably because of the dysfunction of renal tubular epithelial cells due to increased proteins in the tubular lumen. ${ }^{[69]}$ Likewise, Wang et al. in China showed $\mathrm{Cr}$ (VI) concentration and NAG of urine samples in exposed $\mathrm{Cr}$ workers was significantly higher than controls. ${ }^{[70]}$

A study by Kalantari and Farokhi on 24 platers from 8 workplaces in Isfahan showed that exposure to $\mathrm{Cr}$ VI in these workers was higher than the authorized amount by the National Institute for Occupational Safety and Health (NIOSH) for air in workplaces, and workers who dealt with $\mathrm{Cr}$ VI directly and worked near tubs of $\mathrm{Cr}$, were exposed to $\mathrm{Cr} \mathrm{VI}$ more than other workers working in other parts in the same industry. ${ }^{[71]}$ Likewise, Gibb et al. in USA showed a strong dose-response relation between cumulative exposure to $\mathrm{Cr}$ VI in a chromate production plant and lung cancer. ${ }^{[56]}$

\section{Conclusions}

In industrial jobs, including welding, plating and cement, $\mathrm{Cr}$ VI concentrations were higher in workers who directly dealt with $\mathrm{Cr}$ than others employed in the same industry. Therefore, in Iran, exposure to Cr VI should be under surveillance in these occupations and unnecessary exposure should be prevented.

In the general population of Iran, people who lived near a factory or an industrial center, and who had taken dental treatments with orthodontic appliances, had higher concentration of $\mathrm{Cr}$ than others. Likewise, in several studies, $\mathrm{Cr}$ III deficiency was investigated in relation to diabetes type 2 and shows the probable necessity of this element in glucose metabolism.

\section{CONFlicts OF INTEREST Disclosure}

The authors have no competing interests.

\section{REFERENCES}

[1] LENNTECH. Chromium - Cr (Chemical properties of chromium Health effects of chromium - Environmental effects of chromium). [cited 2016 Nov 24]; Available from: http://www. lenntech.com /periodic/elements/cr.htm

[2] CDC/ATSDR. Chromium Toxicity-Agency for Toxic Substances and Disease Registry Case Studies in Environmental Medicine (CSEM) (Pdf) Toxicological Profile for Asbestos. Update. Agency for Toxic Substances and Disease Registry. 2008. [cited 2016 Nov 24]; 67]. Available from: http://www.atsdr.cdc.gov/csem/chromium/ docs/chromium.pdf

[3] Agency-forToxic-Substances-and-Disease-Registry(ATSDR). ToxGuide for Chromium-Cr. 2012. [cited 2016 Nov 24]; Available from: http://www.atsdr.cdc.gov/toxguides/toxguide-7 .pdf

[4] Tchounwou PB, et al. Heavy metal toxicity and the environment, in Molecular, clinical and environmental toxicology. Springer; 2012. 133-164 p. PMid:22945569 https://doi.org/10.1007/978-3 -7643-8340-4_6

[5] International-Agency-for-Research-on-Cancer. List of Classifications. IARC Monographs on the Evaluation of Carcinogenic Risks to Hu- mans [cited 2016 Nov 24]; Available from: http://monographs .iarc.fr/ENG/Classification/latest_classif.php

[6] International-Agency-for-Research-on-Cancer, IARC Monographs on the evaluation of the carcinogenic risk of chemicals in humans: Chromium, Nickel and Welding. 1990; 49: 677.

[7] Independent-Environmental-Technical-Evaluation-Group(IETEG), Chromium (VI) Handbook. CRC Press; 2005.

[8] MedicalNewsToday. What are the health benefits of chromium? 2015 [cited 2017 May 19]; Available from: http://www.medicalnew stoday.com/articles/288177.php

[9] Occupational-Safety-and-Health-Administration. Table Z-1-Limits for Air Contaminants. [cited 2016 Nov 24]; Available from: https : //www.osha.gov/pls/oshaweb/owadisp.show_document?p _table=STANDARDS\&p_id=9992\&p_text_version=FALSE

[10] Amini F, et al. Effects of fixed orthodontic treatment using conventional versus metal-injection molding brackets on salivary nickel and chromium levels: a double-blind randomized clinical trial. The European Journal of Orthodontics. 2014.

[11] Amini F, et al. Effects of fixed orthodontic treatment on hair nickel and chromium levels: a 6-month prospective preliminary 
study. Biological Trace Element Research, 2015. 164(1): 12-17. PMid:25503776 https ://doi .org/10.1007/s12011-014-018 8-0

[12] Amini F, et al. Metal ion release from fixed orthodontic appliances - an in vivo study. The European Journal of Orthodontics. 2012; 34(1): 126-130. PMid:21303810 https://doi.org/10.1 093/ejo/cjq181

[13] Amini F, et al. Effects of fixed orthodontic treatment on nickel and chromium levels in gingival crevicular fluid as a novel systemic biomarker of trace elements: A longitudinal study. American Journal of Orthodontics and Dentofacial Orthopedics. 2016; 149(5): 666-672. PMid:27131248 https://doi.org/10.1016/j.ajodo.2015.1 0.023

[14] Masjedi MK, et al. Effects of fixed orthodontic treatment using conventional (two-piece) versus metal injection moulding brackets on hair nickel and chromium levels: a double-blind randomized clinical trial. The European Journal of Orthodontics. 2016.

[15] Amini F, Rakhshan V, Mesgarzadeh N. Effects of long-term fixed orthodontic treatment on salivary nickel and chromium levels: a 1-year prospective cohort study. Biological Trace Element Research. 2012; 150(1-3): 15-20. PMid:22644664 https://doi .org/10.1007/s1 2011-012-9457-y

[16] Amini F, et al. In vivo study of metal content of oral mucosa cells in patients with and without fixed orthodontic appliances. Orthodontics \& Craniofacial Research. 2008; 11(1): 51-56. PMid:18199080 https://doi.org/10.1111/j.1601-6343.2008.00414.x

[17] Arash V, et al. Measurement of iron, magnesium and chromium concentrations in the saliva of the patients undergoing fixed orthodontic treatment. Caspian Journal of Dental Research. 2012; 1(1): 27-31.

[18] Yassaei S, et al. Nickel and chromium levels in the saliva of patients with fixed orthodontic appliances. ORTHODONTICS: The Art \& Practice of Dentofacial Enhancement. 2013; 14(1): 76-81. https://doi.org/10.11607/ortho.810

[19] Amini F, et al. The effect of stress on salivary metal ion content in orthodontic patients. Biological Trace Element Research. 2013; 155(3): 339-343. PMid:24026442 https://doi.org/10.1007/ s12011-013-9812-7

[20] Amini F, et al. Effect of Stress on Salivary Ion Content in Orthodontic Patients: a pilot study. Journal of Research in Dental Sciences. 2014; 11(2): $65-70$ [persian].

[21] Nourmohammadi L, et al. Zinc, copper, chromium, manganese and magnesium levels in serum and hair of insulin-dependent diabetics. Archives OF Iranian Medicine. 2000; 2(3): 1-5.

[22] Basaki M, et al. Zinc, copper, iron, and chromium concentrations in young patients with type 2 diabetes mellitus. Biological Trace Element Research. 2012; 148(2): 161-164. PMid:22351156 https://doi.org/10.1007/s12011-012-9360-6

[23] Rafiei R, et al. Chromium level in prediction of diabetes in prediabetic patients. Advanced Biomedical Research. 2014; 3: 235 PMid:25538921 https://doi.org/10.4103/2277-9175.1457 37

[24] Sabouri S, et al. The relationship between serum level of chromium and serum malondialdehyde in patients with type II diabetes. Journal of Shahrekord University of Medical Sciences. 2010; 12(2): 1-7 [persian].

[25] Palizban A, et al. The investigation of blood concentration of Vanadium and Chromium in metabolic syndrome patients with and without type 2 diabetes. Journal of Lorestan University of Medical Sciences. 2016; 18(1): 74-81 [persian].

[26] Parsaeian N, et al. Serum Concentration of Chromium in Type 2 patients who referred to Diabetes Center of Shahid Sadoughi University of Medical Sciences, Yazd. Journal of Shahid Sadoughi University of Medical Sciences. 2003; 10(4): 66-71 [persian].

[27] Tadayon F, Tehrani MS, Nia SR. Determination of toxic and essential elements in the scalp hair of patients with type 2 diabetes. Academic Research International. 2012; 2(3): 11-16.

[28] Abdolsamadi H, et al. Comparative Evaluation of Chromium and Cadmium in Gestational diabetes and healthy pregnant women. Iranian Journal of Endocrinology and Metabolism. 2012; 13(6): 666-673 [persian].

[29] Nasli-Esfahani E, et al. Trace element analysis of hair, nail, serum and urine of diabetes mellitus patients by inductively coupled plasma atomic emission spectroscopy. Journal of Diabetes and Metabolic Disorders. 2011; 10: 5.

[30] YousefiRad E, Mohtadinia J, Aliasgarzadeh A. The relationship between dietary intake and serum levels of chromium, lipid profile and $\mathrm{HbA1c}$ in patients with type D. Journal of Lorestan University of Medical Sciences. 2009; 11(2): 43-55.

[31] Makhlough A, et al. Comparing the Levels of Trace Elements in Patients With Diabetic Nephropathy and Healthy Individuals. Nephrourology Monthly. 2015; 7(4). PMid:26539418 https ://doi .org/ 10.5812/numonthly. 28576

[32] Tadayon F, et al. Relationship between the level of zinc, lead, cadmium, nickel and chromium in hair of people with diabetes in E3S Web of Conferences. 2013. EDP Sciences. https://doi.org/10 .1051/e3sconf/20130141012

[33] Mazdak H, et al. Study of Serum level of Chrome in Bladder Cancer Patients. Armaghane Danesh. 2008; 13(3): 124-130 [persian].

[34] Mozaffarinia K, Yazdekhasti F, Abtahi H. The Levels of Serum Chromium and Manganese in Patients with Idiopathic Tinnitus Compared with Healthy Individuals J Isfahan Med Sch. 2015; 33(340): 992-998 [persian].

[35] Alirezaee M, et al. Epidemiological study of trace elements (Iron, Zinc, Copper, Chromium and Cobalt) in nails of sexually premature and matured boys. Iranian Journal of Biology. 2013; 26(1): 136-144 [persian].

[36] Nazarpour S, Teimoori L, Teimoori S. Cadmium and Chrome Concentrations in Human Milk. Journal of Knowledge \& Health. 2013; 9(1): 21-29 [persian].

[37] Hashemian M, et al. The Nail as a Biomonitor of Trace Element Status in Golestan Cohort Study. Middle East Journal of Digestive Diseases. 2016; 8(1): 19. PMid:26933477 https ://doi.org/10 $.15171 / \mathrm{mejdd} .2016 .02$

[38] Eskandary H, Nowbari N. Serum concentration of chromium in head injury patients. Archives of Iranian Medicine. 2002; 5(3): 162.

[39] Golbabaei F, et al. Assessment of welders exposure to carcinogen metals from manual metal arc welding in gas transmission pipelines, iran. Iranian Journal of Public Health. 2012; 41(8): 61. PMid:23113226

[40] Pournourmohammadi S, et al. Study on the oxidative stress status among cement plant workers. Human \& Experimental Toxicology. 2008; 27(6): 463-469. PMid:18784198 https://doi.org/10.1 $177 / 0960327108094956$

[41] Golbabaei F, et al. Feasibility of biological monitoring for evaluating of exposure to Cr6 in Electroplating workshops Journal of School of Public Health and Institute of Public Health Research. 2007; 5(3): 15-22 [persian].

[42] Krejpcio Z. Essentiality of chromium for human nutrition and health. Polish Journal of Environmental Studies. 2001; 10(6): 399-404.

[43] Cefalu WT, Hu FB. Role of chromium in human health and in diabetes. Diabetes Care. 2004; 27(11): 2741-2751. PMid:15505017 https://doi.org/10.2337/diacare.27.11.2741 
[44] Anderson RA. Effects of chromium on body composition and weight loss. Nutrition Reviews. 1998; 56(9): 266-270. PMid:9763876 https://doi.org/10.1111/j.1753-4887.1998.tb01763.x

[45] Anderson RA. Recent advances in the clinical and biochemical effects of chromium deficiency. Progress in Clinical and Biological Research. 1992; 380: 221-234.

[46] Amr N, Abdel-Rahim HE. The effect of chromium supplementation on polycystic ovary syndrome in adolescents. Journal of Pediatric and Adolescent Gynecology. 2015; 28(2): 114-118. PMid:25850593 https://doi.org/10.1016/j.jpag.2014.05.005

[47] Gale-Encyclopedia-of-Alternative-Medicine. Chromium. 2017 [cited 2017 May 13]; Available from: http: //www.encyclopedia.com/science-and-technology/ chemistry/compounds-and-elements/periodic-table-e lements-chromium\#3400900112

[48] ATSDR. What Are the Physiologic Effects of Chromium Exposure? 2013 [cited 2017 March 28]; Available from: https://www. atsd r.cdc.gov/csem/csem . asp?csem $=10 \& p o=10$

[49] Wilbur S, et al. Health effects, in Toxicological Profile for Chromium. ATSDR: USA. 2012.

[50] Jacobs JA, Testa SM. Overview of chromium (VI) in the environment: background and history. CRC Press: Boca Raton, FL. 2005.

[51] Vimercati L, et al. Environmental exposure to arsenic and chromium in an industrial area. Environmental Science and Pollution Research. 2017; 24(12): 11528-11535. PMid:28321698 https://doi.org/ $10.1007 / \mathrm{s} 11356-017-8827-6$

[52] Lukaski HC. Magnesium, zinc, and chromium nutriture and physical activity. The American Journal of Clinical Nutrition. 2000; 72(2): 585s-593s. PMid: 10919964

[53] Interdonato $\mathrm{M}$, et al. Levels of heavy metals in adolescents living in the industrialised area of Milazzo-Valle del Mela (northern Sicily). Journal of Environmental and Public Health. 2014.

[54] Haase GM, et al. Antioxidant micronutrient impact on hearing disorders: concept, rationale, and evidence. American Journal of Otolaryngology. 2011; 32(1): 55-61. PMid:20015808 https ://doi or $\mathrm{g} / 10.1016 / \mathrm{j}$. amjoto. 2009.09.002

[55] Pechova, A. and L. Pavlata, Chromium as an essential nutrient: a review. VETERINARNI MEDICINA-PRAHA. 2007; 52(1): 1.

[56] Gibb HJ, et al. Lung cancer among workers in chromium chemical production. American Journal of Industrial Medicine. 2000; 38(2): 115-126. https://doi.org/10.1002/1097-0274(200008) 38: 2<115: :AID-AJIM1>3.0.CO;2-Y

[57] Ciorba A, et al. Pathogenesis of Tinnitus: Any Role for Oxidative Stress? Int. Adv. Otol. 2013; 9(2): 249-254.

[58] Neri S, et al. Tinnitus and oxidative stress in a selected series of elderly patients. Archives of Gerontology and Geriatrics. 2002; 35: 219223. https://doi.org/10.1016/S0167-4943(02)00137-1
[59] Lai MH. Antioxidant effects and insulin resistance improvement of chromium combined with vitamin $\mathrm{C}$ and $\mathrm{E}$ supplementation for type 2 diabetes mellitus. Journal of Clinical Biochemistry and Nutrition. 2008; 43(3): 191-198. PMid:19015754 https://doi.org/10.3 $164 /$ jcbn. 2008064

[60] Anderson RA, et al. Potential antioxidant effects of zinc and chromium supplementation in people with type 2 diabetes mellitus. Journal of the American College of Nutrition. 2001; 20(3): 212218. PMid:11444416 https://doi.org/10.1080/07315724.2 001.10719034

[61] Santonen T. Inorganic chromium (III) compounds. World Health Organization. 2009.

[62] Ağaoğlu G, et al. Nickel and chromium levels in the saliva and serum of patients with fixed orthodontic appliances. The Angle Orthodontist. 2001; 71(5): 375-379. PMid:11605871

[63] Singh DP, et al. Estimation of nickel and chromium in saliva of patients with fixed orthodontic appliances. World Journal of Orthodontics. 2008; 9(3). PMid: 18834002

[64] Kocadereli I, et al. Salivary nickel and chromium in patients with fixed orthodontic appliances. The Angle Orthodontist. 2000; 70(6): 431-434. PMid:11138646

[65] Kazi TG, et al. Copper, chromium, manganese, iron, nickel, and zinc levels in biological samples of diabetes mellitus patients. Biological Trace Element Research. 2008; 122(1): 1-18. PMid:18193174 https://doi.org/10.1007/s12011-007-8062-y

[66] Ghosh D, et al. Role of chromium supplementation in Indians with type 2 diabetes mellitus. The Journal of Nutritional Biochemistry. 2002; 13(11): 690-697. https ://doi.org/10.1016/S0955-286 3(02) 00220-6

[67] OSHA. Health Effects of Hexavalent Chromium. 2006 [cited 2017 May 19]; Available from: https://www.osha.gov/0shDoc/da ta_General_Facts/hexavalent_chromium.pdf

[68] NationalCancerInstitute. Hexavalent Chromium Compounds. 2015 [cited 2017 May 20]; Available from: https://www . cancer.gov/about-cancer/causes-pre vention/risk/substances/chromium

[69] Bazzi C, et al. Urinary $\mathrm{N}$-acetyl- $\beta$-glucosaminidase excretion is a marker of tubular cell dysfunction and a predictor of outcome in primary glomerulonephritis. Nephrology Dialysis Transplantation. 2002; 17(11): 1890-1896. https://doi.org/10.1093/ndt/17 .11 .1890

[70] Wang X, et al. Chromium-induced early changes in renal function among ferrochromium-producing workers. Toxicology. 1994; 90(12): 93-101. https://doi .org/10.1016/0300-483X (94) 90208 $-9$

[71] Kalantari A, Farokhi Z. Exposure of plating workers to hexavalent chromium in workplaces of Isfahan in 2004, in Forth Congress of Occupational Health and Safty in Iran, 4-6 October. 2004 [persian]: Hamadan, Iran. 